\title{
HIERARCHIES OF NUMBER-THEORETIC PREDICATES
}

\author{
S. C. KLEENE
}

The existence of hierarchies of point sets in analysis has long been familiar from the work of Borel and Lusin. The study of the hierarchies in number theory which we consider here began with a theorem presented to the Society in 1940 and published in [12]. These hierarchies have applications in foundational investigations, but we shall be concerned here with the exploration of their structure (using classical logic). We shall survey the previous results from the beginning, and conclude with a few new ones. We have endeavored to make the exposition complete enough so that the layman in this field can get the gist of the arguments without consulting the references.

1. Recursive functions and predicates. By a number-theoretic function (predicate) we mean a function, of independent variables ranging over the natural numbers $0,1,2, \cdots, x, x+1, \cdots$, taking natural numbers (propositions, true or false) as values.

By general recursive functions (predicates) we mean ones whose values can be computed (decided) by ideal computing machines not limited in their space for storing information. A theory of such machines was given by Turing [30] and in less detail by Post [24] (also cf. [16, Chapter XIII]). The general recursive functions can also be described as those whose values can be expressed by equations derivable formally from "recursion equations" defining the functions, in a sense first formulated precisely by Gödel [9] who built on a suggestion of Herbrand's (also cf. Church's [3], and our [10;12;16, Chapter XI]). A general recursive predicate $P\left(x_{1}, \cdots, x_{n}\right)$ is then one whose representing function $\phi\left(x_{1}, \cdots, x_{n}\right)$ ( $=0$ or 1 according as $P\left(x_{1}, \cdots, x_{n}\right)$ is true or false) is general recursive.

The computation of a value of a general recursive function may involve a search through the natural numbers for the least one $y$ with a given property without a bound for such a $y$ having already been computed. By allowing such searches also when they may not terminate, we obtain an extension of the class of the general recursive functions to the partial recursive functions, which need not be defined for all sets of arguments $[11 ; 12 ; 16$, Chapter XII $]$. By disallowing such

An address delivered before the Chicago meeting of the Society on April 30, 1954, by invitation of the Committee to Select Hour Speakers for Western Sectional Meetings; received by the editors July 3, 1954 . 
searches altogether, and requiring the functions to be generated, starting with the variables, 0 and $x+1$, by "primitive recursions", in which the function value for an argument $y+1$ is given in terms of that for $y$, and substitutions, we obtain a specialization of the general recursive functions to the primitive recursive functions $[8 ; 10 ; 12 ; 16$, Chapter IX]. Predicates are partial (primitive) recursive, if their representing functions are such.

Since a particular partial recursive function is defined by a machine or by a system of equations, which is a finite object, the class of the partial (including the general) recursive functions is countable. It is in fact possible to assign numbers many-one to the partial recursive functions so that, given a number of a function and a set of arguments for it, we can compute the value (if defined). We state this result (with additional details) as a theorem.

I. For each $n \geqq 0$ : There is a fixed partial recursive function $\Phi_{n}\left(z, x_{1}, \cdots, x_{n}\right)$ such that, to any partial recursive function $\phi\left(x_{1}, \cdots, x_{n}\right)$ there is a number $e$ (called a Gödel number of $\phi$ ) for which $\phi\left(x_{1}, \cdots, x_{n}\right) \simeq \Phi_{n}\left(e, x_{1}, \cdots, x_{n}\right)$. In fact: $\Phi_{n}\left(z, x_{1}, \cdots, x_{n}\right)$ $\simeq U\left(\mu y T_{n}\left(z, x_{1}, \cdots, x_{n}, y\right)\right)$ where $U$ is a primitive recursive function, $T_{n}$ is a primitive recursive predicate, and $\mu y$ means "the least $y$ such that". (The normal form theorem.)

We write $\simeq$ instead of $=$ in equations between partial recursive functions to indicate that they can hold by both sides being undefined (e.g. $\phi(x)=\phi(x)+1$ is absurd, but $\phi(x) \simeq \phi(x)+1$ merely implies that $\phi(x)$ is undefined). The numbers $e$ we call "Gödel numbers", because we obtained them in $[10 ; 11 ; 12 ; 16$, pp. 288, 330, 340] by applying essentially Gödel's method of numbering [8] to the systems of equations.

II. For each $m, n \geqq 0$ : There is a primitive recursive function $S_{n}^{m}\left(z, y_{1}, \cdots, y_{m}\right)$ such that, if $e$ is a Gödel number of $\phi\left(y_{1}, \cdots, y_{m}\right.$, $\left.x_{1}, \cdots, x_{n}\right)$ as a function of all $m+n$ variables, then for each $m$ fixed numbers $y_{1}, \cdots, y_{m}, S_{n}^{m}\left(e, y_{1}, \cdots, y_{m}\right)$ is a Gödel number of $\phi\left(y_{1}, \cdots, y_{m}, x_{1}, \cdots, x_{n}\right)$ as a function of the $n$ remaining variables $x_{1}, \cdots, x_{n}$.

We trust this will appear plausible. (For details, cf. $[11 ; 16$, p. 342 (erratum, 1952 printing, 1. 7 from below, replace " $d * \ldots$ " by " $d *[2 \exp \cdots] ")]$.)

III. Given any partial recursive function $\chi\left(z, x_{1}, \cdots, x_{n}\right)$, there is a Gödel number $e$ of $\chi\left(e, x_{1}, \cdots, x_{n}\right)$. (The recursion theorem.)

Let $f$ be a Gödel number of $\chi\left(S_{n}^{1}(y, y), x_{1}, \cdots, x_{n}\right)$, and let $e=S_{n}^{1}(f, f)$.

By III, we can introduce a partial recursive function $\phi$ whose value 
$\phi\left(x_{1}, \cdots, x_{n}\right)$ is expressed partial recursively in terms of a Gödel number $e$ of $\phi$ itself and the arguments $x_{1}, \cdots, x_{n}$; i.e. we can put $\phi\left(x_{1}, \cdots, x_{n}\right) \simeq \chi\left(e, x_{1}, \cdots, x_{n}\right)$ for $\chi$ a given partial recursive function and $e$ a Gödel number of $\phi$. Sometimes the form of $\chi$ will then enable us to infer that $\phi$ is general or even primitive recursive. $[11 ; 16$, pp. $352 \mathrm{ff}$.$] .$

2. The arithmetical hierarchy. Most predicates used in elementary number theory can be expressed by starting with general recursive predicates and applying the logical operations of the restricted predicate calculus, i.e. the propositional connectives \& (and), $\vee$ (or), (not) and $\rightarrow$ (implies), and the quantifiers, generality ( $x$ ) (for all $x$ ) and existential (Ex) (there exists an $x$ such that). We call these predicates arithmetical, following Gödel who introduced them a little differently $[8 ; 9 ; 16$, pp. 239,285$]$.

Let $p_{i}$ be the $i$ th prime number, counting 2 as the 0 th. Let $(a)_{i}$ $=\left\{\right.$ the exponent of $p_{i}$ in $a$ as a product of powers of distinct primes, if $a \neq 0 ; 0$, if $a=0\}\left[16\right.$, p. 230]. Then $\left(x_{0}\right) \cdots\left(x_{m}\right) A\left(x_{0}, \cdots, x_{m}\right)$ $\equiv(x) A\left((x)_{0}, \cdots,(x)_{m}\right)$, and similarly with existential quantifiers.

IV. Every arithmetical predicate $P(a)$ is expressible in one of the forms

(a) $R(a)$

$$
\begin{aligned}
(E x) R(a, x) & (x)(E y) R(a, x, y) & (E x)(y)(E z) R(a, x, y, z) & \cdots \\
(x) R(a, x) & (E x)(y) R(a, x, y) & (x)(E y)(z) R(a, x, y, z) & \cdots
\end{aligned}
$$

where the $R$ for each form is general recursive.

One of these forms will be obtained, starting with an expression for $P(a)$ as an arithmetical predicate, by first advancing the quantifiers to the front, and then contracting consecutive like quantifiers as just indicated $[12 ; 16$, p. 285]. For brevity we are stating IV-VIII for one variable $a$, but they hold for $x_{1}, \cdots, x_{n}$ instead $(n \geqq 0$ for IV, V, VIII, $n>0$ for VI, VII).

$\mathrm{V}$. Given any general recursive predicate $R(a, x)$, there is a number $f$ such that $(E x) R(a, x) \equiv(E x) T_{1}(f, a, x)$. Similarly for the other quantified forms of (a). (The enumeration theorem.)

Let $f$ be a Gödel number of $\mu x R(a, x)$. This function is defined for given $a$, by definition if and only if $(E x) R(a, x)$; by $f$ being its Gödel number if and only if $(E x) T_{1}(f, a, x)$. Similarly $(E x)(y) R(a, x, y)$ $\equiv(E x)(y) \bar{T}_{2}(g, a, x, y)$ when $g$ is a Gödel number of $\mu y \bar{R}(a, x, y)$. $[12 ; 16$, p. 281$]$. Incidentally, $\mathrm{V}$ shows that the same predicates are expressible in a given quantified form of (a) with a primitive as with a general recursive $R$.

VI. To each of the quantified forms of (a) there is a respective predicate 
$\begin{array}{llll}(E x) T_{1}(a, a, x) & (x)(E y) T_{2}(a, a, x, y) & (E x)(y)(E z) T_{3}(a, a, x, y, z) & \ldots\end{array}$

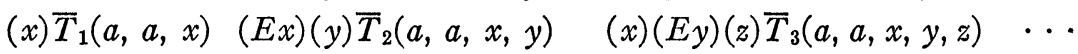

of that form which is not expressible in the dual form, a fortiori not in any of the forms with fewer quantifiers. (The hierarchy theorem.)

Given a recursive $R(a, x)$, the $f$ of $\mathrm{V}$ is a value of $a$ for which $(x) \bar{T}_{1}(a, a, x) \not \equiv(E x) R(a, x)[12 ; 16$, p. 283].

By a complete 1-place predicate of a certain form (or belonging to a certain class) we mean a predicate $C(a)$ of the form and such that to each predicate $P(a)$ of the form there is a recursive function $\theta(a)$ (actually primitive recursive in all our examples) for which $P(a)$ $\equiv C(\theta(a))$.

VII. The predicate $(E x) T_{1}(a, a, x)$ of VI is a complete predicate of the respective form $(E x) R(a, x)$ of (a). Similarly for the other quantified forms of (a).

Let $e$ be a Gödel number of $\mu x R(a, x)$ as a function of $a, t$ (in Church's $\lambda$-notation $[16$, p. 34], of the function $\lambda a t \mu x R(a, x))$. Then $S_{1}^{1}(e, a)$ is one of $\lambda t \mu x R(a, x)$, which for any $t$ is defined if and only if $(E x) R(a, x)$. So $(E x) R(a, x) \equiv(E x) T_{1}\left(S_{1}^{1}(e, a), t, x\right)$, whence substituting $S_{1}^{1}(e, a)$ for $t,(E x) R(a, x) \equiv C(\theta(a))$ for $C(a) \equiv(E x) T_{1}(a, a, x)$ and $\theta(a)=S_{1}^{1}(e, a)$. ([16, p. 343]. A different $C(a)$ was used by Post [25], and by the author in lectures in 1941; cf. [19, Footnote 8].)

VIII. The general recursive predicates $R(a)$ are exactly those expressible in both the 1-quantifier forms of (a). (Analog of Souslin's theorem.)

For $R(a) \equiv(E x) R(a) \equiv(x) R(a)$. Conversely, if $R(a) \equiv(E x) R(a, x)$ $\equiv(x) S(a, x)$ with $R$ and $S$ recursive, then $\bar{R}(a) \equiv(E x) \bar{S}(a, x)$, and by first calculating $\mu x(R(a, x) \bigvee \bar{V}(a, x))$ we can decide $R(a)$. ([16, p. 284]. The theorem was given by the author [12], by Post [25], and by Mostowski [22] who noted that it is analogous to a theorem of Souslin on Borel and analytic sets.)

3. Relative recursiveness. Instead of a machine for computing a function $\phi\left(x_{1}, \cdots, x_{n}\right)$ outright, we can imagine one which would compute $\phi$, if there were supplied to the machine values of a function $\psi(x)$ on demand, i.e. for arguments arising in the computation. (In this paper the $\psi$ shall be a completely defined function.) This idea leads us to functions and predicates general (partial, primitive) recursive in $\psi$, and predicates arithmetical in $\psi$. ([16, pp. 275, 326, 224, $291]$. In terms of machines, the idea appeared in a special setting in Turing [31, §4]; in terms of general recursiveness, in [12].)

We can obtain relativized versions of the above theorems simply by modifying the proof of I to suppose values of $\psi$ available in the com- 
putation of $\phi$. However it is useful to observe that at any stage of the computation only a finite number of values of $\psi$ can have been used. So the computation can be arranged to involve a search in which at the $y$ th stage only the first $y$ values $\psi(0), \cdots, \psi(y-1)$ of $\psi$ have been made available. These $y$ values can be represented by either of the two numbers

$$
\bar{\psi}(y)=\prod_{x<y} p_{x}^{\psi(x)+1}, \quad \tilde{\psi}(y)=\prod_{x<y} p_{x}^{\psi(x)} .
$$

Note that $y=\operatorname{lh}(\bar{\psi}(y))$ with $\mathrm{lh}$ primitive recursive $[16$, p. 230].

IX. Theorems I-VIII hold when in place of the notions 'recursive', ' $\Phi_{n}$ ', 'Gödel number', ' $T_{n}$ ', ' $S_{n}^{m}$, 'arithmetical', '1-quantifier forms' we use 'recursive in $\psi$ ', ' $\Phi_{n}^{\psi}$ ', 'Gödel number from $\psi$ ', ' $T_{n}^{\psi}$ ', ' $S_{n}^{m, 1}$ ', 'arithmetical in $\psi$ ', '1-quantifier forms relative to $\psi$ ', respectively, except that $U, S_{1}^{m, 1}$ are still primitive recursive (call them then $\mathrm{I}^{*}$-VIII*). Moreover $T_{n}^{\psi}\left(z, x_{1}, \cdots, x_{n}, y\right) \equiv T_{n}^{1}\left(\bar{\psi}(y), z, x_{1}, \cdots, x_{n}\right)$ where $T_{n}^{1}$ is primitive recursive.

We have stated IX for one (completely defined) function $\psi$ of one variable (hence the superscript " $\psi$ " or sometimes " 1 " for the number of its variables). We could instead have a (completely defined) predicate $Q$, where $\psi$ is its representing function; or we could use any finite list $\Psi$ of (completely defined) functions and predicates $[16$, p. 292].

$\mathrm{X}$. There is a number $g$ such that, if $A(a)$ is recursive in $B(a)$ with Gödel number e, then $(E x) T_{1}^{A}(a, a, x)$ is recursive in $(E x) T_{1}^{B}(a, a, x)$ with Gödel number $S_{1}^{1,1}(g, e)$.

For any 1-place predicate $B$ and number $e$, let $B_{e}$ be the 1-place function partial recursive in $B$ with Gödel number $e$. By the method of proof of VII*, there is a primitive recursive function $\theta$, independent of $B$, such that $(E x) T_{1}^{B_{e}}(a, a, x) \equiv(E x) T_{1}^{B}(\theta(e, a), \theta(e, a), x)$ for values of $B, e, a$ which make $\lambda x T_{1}^{B_{e}}(a, a, x)$ completely defined. Let $g$ be a uniform Gödel number from $P$ (i.e. one independent of $P$ ) of $\lambda e a P(\theta(e, a))$. [19, beginning 4.1]. A X* holds, e.g. with " $Q, B(a)$ ", " $T_{1}^{Q, A}$ ", " $T_{1}^{Q, B}$ " in place of " $B(a)$ ", “ $T_{1}^{A}$ ", " $T_{1}^{B}$ ".

The relation ' $P$ is general recursive in $Q$ ' is reflexive and transitive $[16$, p. 275]. Hence ' $P$ is general recursive in $Q$ and vice versa' is reflexive, symmetric and transitive. Post in $[25 ; 26]$ associates with each predicate $P$ a degree so that $P$ and $Q$ possess the same degree exactly if each is general recursive in the other. The degree of $P$ is less than that of $Q$, if $P$ is recursive in $Q$ but not vice versa. A complete predicate of a given form (cf. VII, VII*) is of maximal degree for predicates of that form. Applying $\mathrm{X}$ in two directions to predi- 
cates $Q_{1}, Q_{2}$ of the same degree, the degree of $(E x) T_{1}^{Q}(a, a, x)$ depends only on the degree of $Q$. The structure of the system of the degrees is discussed in detail in [19].

The predicates of VI of the two $k+1$-quantifier forms are negations of each other, and hence are of the same degree. But in XII we shall see that they are of higher degree than any predicate expressible with fewer quantifiers. For this we need the following generalization of VIII due to Post [26].

XI. For each $k \geqq 0$ : The predicates general recursive in predicates expressible in the k-quantifier forms of (a) are exactly the predicates expressible in both the $k+1$-quantifier forms. (Post's theorem.)

That a predicate expressible in both $k+1$-quantifier forms is recursive in $k$-quantifier predicates is proved for $k>0$ essentially as before for $k=0$. The proof of the converse is a little too detailed to be sketched here; it can be based on the special form of $\mathrm{V}^{*}$ in terms of $\bar{\psi}(y)$ or $\tilde{\psi}(y)\left[16\right.$, p. 293]. An XI* referring similarly to $\mathrm{IV}^{*}$ is obtained by reading "forms relative to $Q$ " for "forms".

Let $N_{0}(a) \equiv L_{0}(a) \equiv a=a$. Let the predicates in the upper row of VI be abbreviated $N_{1}(a), N_{2}(a), N_{3}(a), \cdots$ Let $L_{k+1}(a) \equiv(E x)$ $T_{1}^{L_{k}}(a, a, x)$.

XII. For each $k \geqq 0: N_{k+1}$ is expressible in a $k+1$-quantifier form of (a), is of maximal degree for predicates so expressible, and is of higher degree than $N_{k}$. Similarly with ' $L$ ' in place of ' $N$ '. (The strengthened hierarchy theorem.)

The proof with ' $N$ ' is immediate from VII, XI and VI; with ' $L$ ' it follows thence, using induction on $k$ and XI, VII*. A XII* with ' $N^{Q}$ ' and ' $L^{Q}$ ' is based similarly on $\mathrm{VI}^{*}, \mathrm{VII}^{*}, \mathrm{XI}^{*}\left(N_{0}^{Q}(a) \equiv L_{0}^{Q}(a)\right.$ $\equiv Q(a) ; N_{1}^{Q}, N_{2}^{Q}, N_{3}^{Q}, \cdots$ is the upper row of VI*; $L_{k+1}^{Q}(a) \equiv(E x)$ $\left.T_{1}^{L_{k}^{Q}}(a, a, x)\right)$.

4. The extended arithmetical hierarchy. Kalmár first noticed that nonarithmetical predicates can be defined by induction using quantifiers within the induction step. (Cf. Skolem [27]; we gave a simple example in $\left[12 ; 16\right.$, p. 287].) Let $N(a, k) \equiv N_{k}(a), L(a, k) \equiv L_{k}(a)$. Then $N$ and $L$ are of degree exceeding that of every arithmetical predicate. For each arithmetical predicate is recursive in one of $N_{0}, N_{1}, N_{2}, \cdots$, each of which is recursive in $N$, and the degrees of which are ascending; and similarly with ' $L$ '. $(N$ and $L$ are of the same degree, by [19, Footnote 29].)

The predicate $L(a, k)$ can be contracted to a 1-place predicate $L(a) \equiv L\left((a)_{0},(a)_{1}\right)$ of the same degree. Then by using XII* with $Q(a) \equiv L(a)$ we get a sequence of predicates of degrees ascending from that of $L$. This process can be repeated, so that we obtain 
predicates correlated to various transfinite ordinal numbers, beginning with $L$ correlated to $\omega$.

However for this to make sense we must be careful in our handling of the ordinals. We might correlate to $\omega$ also $L(a, \phi(k))$ where $\phi(k)$ is any other increasing function than $\phi(k)=k$. But then by the next theorem, various choices of $\phi$ would give predicates correlated to $\omega$ of arbitrarily high degrees. (To any function $\psi, \phi(k)=\sum_{x<k} \psi(x)+1$ is an increasing function of the same degree.)

XIII. For any function $\phi(k)$ : The function $\phi(k)$ is general recursive in the predicate $N(a, \phi(k))$, and in the predicate $L(a, \phi(k))$.

Proof. For ' $N$ ', using the actual definition of $T_{n}\left(z, x_{1}, \cdots, x_{n}, y\right)$ $[16$, pp. 278,281$]$, it can be seen that $\mu a N_{k}(a)$ for $k=0$ is 0 , and for $k>0$ is the Gödel number $(\neq 0)$ of a system of equations consisting of a single equation $\mathrm{f}\left(\mathrm{r}_{1}, \cdots, \mathrm{r}_{k}\right)=0$, whence $k$ itself can be read. Thus $k$ (as a function of 0 variables) is partial recursive in $N_{k}$, uniformly in the sense that the indicated method of calculating $k$ from $N_{k}$ is applicable independently of what predicate $N_{k}$ is. On replacing $k$ by $\phi(k)$, this gives the theorem for ' $N$ ' (cf. [16, p. 344]). For ' $L$ ', using the actual definition of $T_{1}^{A}(z, a, x)\left[16\right.$, pp. 290, 291], $L_{0}(0)$ is true, but $L_{k}(a)$ for $k>0$ is only true when $a$ is the Gödel number of a system of equations and hence is $\neq 0$. Also, using VII*, a predicate $A(a)(\equiv(E x) A(a))$ is recursive uniformly in $(E x) T_{1}^{A}(a, a, x)$. So from $L_{k}(a)$ we can find $k$, by asking whether $L_{k}(0)$ is true, if not whether $L_{k-1}(0)$ is true, etc., and counting the questions asked until the answer is affirmative. Thus (using III* for $n=0$ ) $k$ is partial recursive uniformly in $L_{k}$. A XIII* is obtained by reading " $N Q$ ", " $L$ " in place of " $N$ ", " $L$ ", for the case of any $Q(a)$ such that $Q(0)$ is true.

What we do is to handle the ordinals on the basis of recursiveness. Such a theory of constructive ordinals was set up in a series of papers by Church and the author $[5 ; 4 ; 11 ; 13 ; 18]$.

In the version we shall use (the system $S_{3}$ of $[11 ; 13 ; 18]$ ), ordinals are represented by natural numbers. Let $O$ be the class of the natural numbers used, to each $y \in O$ let $|y|$ be the ordinal represented, and let $u<o y$ be the partial ordering relation which holds when $u$ enters into the generation of $y$ as a member of $O$ by the following principles. $1 \in O$ and $|1|=0$. If $y \in O$, then $2^{y} \in O,\left|2^{y}\right|=|y|+1$ and $y<_{o} 2^{y}$. (Thus in particular the finite ordinals $0,1,2, \cdots$ are represented by the numbers $1,2^{1}, 2^{2^{1}}, \cdots$, which we also write $0_{o}, 1_{o}, 2_{o}, \cdots$. When $y \in O$, we often write $2^{y}$ as $y^{*}$.) If $y_{0}, y_{1}, y_{2}, \cdots \in O$, and $y_{0}<_{o} y_{1}<_{o} y_{2}<_{o} \cdots$, and for each $n, y_{n}$ is the value for $n_{o}$ of the partial recursive function with Gödel number $y$ (so $y_{n}=\Phi_{1}\left(y, n_{0}\right)$ ), 
then $3 \cdot 5^{y} \in O,\left|3 \cdot 5^{y}\right|=\lim _{n}\left|y_{n}\right|$, and for each $n, y_{n}<03 \cdot 5^{y}$. These principles, together with the transitivity of $<_{o}$, constitute a simultaneous inductive definition of $O$ and $<_{o}$ and a definition of $|y|$ by induction over $O$.

If $u<o y$, then $|u|<|y|$. (If $u<o y$, then $u, y \in O[13, \S 12 ; 18, \S 20]$.) The ordinals $|y|$ for $y \in O$ constitute a proper segment of Cantor's first and second number classes determined by "the least nonconstructive ordinal" $\omega_{1}$. The predicate $<_{o}$ only partially orders $O$, since for each limit ordinal $\alpha<\omega_{1}$, different ascending sequences with $\lim _{n}\left|y_{n}\right|=\alpha$, different partial recursive functions $\phi$ with $\phi\left(n_{0}\right)=y_{n}$, and different Gödel numbers $y$ of $\phi$, can be chosen. However the natural numbers $u<o$ any fixed natural number $y$ are linearly (and well-) ordered by $<_{o}$ (e.g. $\left.[18, \S 20]\right)$.

Now we define a predicate $H_{y}(a)$ for each $y \in O$, so that $H_{0_{o}}, H_{1_{o}}$, $H_{2_{0}}, \cdots$ will be $L_{0}, L_{1}, L_{2}, \cdots$, and $H_{w}$ will be $L$ when $w$ is a suitable notation for $\omega$ (namely, when $w=3 \cdot 5^{y}$ and $\Phi_{1}\left(y, n_{o}\right)=n_{o}$ ).

We simply put $H_{1}(a) \equiv a=a ; H_{2^{y}}(a) \equiv(E x) T_{1}^{H} y(a, a, x)$ if $y \in O$; and $H_{3 \cdot 5^{y}}(a) \equiv H_{y_{(a)_{1}}}\left((a)_{0}\right)$ if $3 \cdot 5^{y} \in O$ and $y_{n}=\Phi_{1}\left(y, n_{0}\right)$.

XIV. To each $y \in O$, the predicate $H_{y}$ is defined; and for $u<o y, H_{u}$ is of lower degree than $H_{y}$. Moreover, there is a primitive recursive function $\rho(u, y)$ such that, if $y \in O$ and $u \leqq o y$, then $\rho(u, y)$ is a Gödel number of $H_{u}$ from $H_{y}$.

The first sentence is clear by the reasoning applied above to $L_{0}, L_{1}, L_{2}, \cdots$, and $L$. So in particular, for $u \leqq o y, H_{u}$ is recursive in $H_{y}$; examining this recursiveness in detail, we are able using III to obtain a Gödel number for it by a primitive recursive function $\rho(u, y)$ $[17,6.3]$.

Sums $u+o y$ of ordinal notations $u, y \in O$ can be defined by induction so that $+o$ is primitive recursive and $|u+o y|=|u|+|y|[18$, $\S 22]$. In fact $u \leqq o u+o y$ but not always $y \leqq o u+o y$ [18, $\$ 22$ and Footnote 30 ]. Finite sums

$$
\sum_{i<n} o \psi(i)
$$

where $(i)[\psi(i) \in O]$ can then be defined primitive recursively in $\psi$ $[18, \S 23]$.

$\mathrm{XV}$. There is a primitive recursive function $\sigma(u, y)$ such that, if $u, y \in O$, then $H_{y}$ is general recursive in $H_{u+o y}$ with Gödel number $\sigma(u, y)$.

This can be proved similarly to XIV, using X $[17,6.3]$.

Extensions of the arithmetical hierarchy have been discussed by Davis [6; 7] and Mostowski [23], and were known independently to Post and to the author. Davis' extension is as here based on 
$L_{0}, L_{1}, L_{2}, \cdots$; Mostowski's on $N_{0}, N_{1}, N_{2}, \cdots$, besides which he proceeds differently at limit ordinals. After adjusting for this difference, the degrees of the predicates correlated to a given $y \in O$ are the same $[17, \S 9]$. Davis [6] proves that the predicates correlated to different notations for the same ordinal $|y|$ are of the same degree, for $|y|<\omega^{2}$. In [28] Spector will extend this to all $|y|<\omega_{1}$.

5. The analytic hierarchy. When a function $\phi$ or predicate $P$ is (general, partial, primitive) recursive in $\psi$ and the relationship is uniform (i.e. the method of computing $\phi$, or deciding $P$, from $\psi$ is independent of what number-theoretic function $\psi$ is), we can regard $\phi$ as a recursive function, and $P$ as a recursive predicate, of $\psi$ as an independent function variable besides of the number variables [15; 17]. More generally there may be several function variables; we shall usually write them $\alpha, \beta, \gamma, \alpha_{1}, \cdots, \alpha_{n}$, etc. We take them to be variables for 1-place functions, which suffices for present purposes.

If we apply to general recursive predicates of number and function variables the operations of the predicate calculus with quantification only of number variables, we get as before arithmetical predicates except that now they may have function variables. If we apply the operations of the predicate calculus with quantification of function variables also allowed, we get analytic predicates. If their only (free) variables are number variables, they come under the subject of this paper.

The analytic predicates were studied in [17], where we began by seeing to what extent the theory could be developed analogously to [12].

First, by exploring the possible permutations and contractions of number and function quantifiers together, we get an analog of IV.

XVI. Every analytic predicate $P(a)$ is expressible in one of the forms

$$
\begin{array}{llr}
A(a) & (\alpha)(E x) R(a, \alpha, x) & (E \alpha)(\beta)(E x) R(a, \alpha, \beta, x) \\
& (E \alpha)(x) R(a, \alpha, x) & (\alpha)(E \beta)(x) R(a, \alpha, \beta, x)
\end{array}
$$

where $A$ is arithmetical and each $R$ is general recursive. Equivalently these forms can be written respectively

$$
\begin{array}{rr}
(\alpha) A(a, \alpha) & (E \alpha)(\beta) A(a, \alpha, \beta) \\
(E \alpha) A(a, \alpha) & (\alpha)(E \beta) A(a, \alpha, \beta)
\end{array}
$$

where each $A$ is arithmetical.

The first and third of the next theorems follow from $\mathrm{V}^{*}$ and VII* by quantification of function variables; the second from the first as VI from V. 
XVII. Given any general recursive predicate $R(a, \alpha, x)$, there is a number $f$ such that $(\alpha)(E x) R(a, \alpha, x) \equiv(\alpha)(E x) T_{1}^{\alpha}(f, a, x) \equiv(\alpha)$ $(E x) T_{1}^{1}(\bar{\alpha}(x), f, a)$. Similarly for the other quantified forms of $(\mathrm{b})$.

XVIII. To each of the quantified forms of (b) there is a respective predicate

$$
\begin{array}{rrr}
(\alpha)(E x) T_{1}^{\alpha}(a, a, x) & (E \alpha)(\beta)(E x) T_{1}^{\alpha, \beta}(a, a, x) & \ldots \\
(E \alpha)(x) \bar{T}_{1}^{\alpha}(a, a, x) & (\alpha)(E \beta)(x) \bar{T}_{1}^{\alpha, \beta}(a, a, x) & \ldots
\end{array}
$$

of that form which is not expressible in the dual form, a fortiori not in any of the forms with fewer quantifiers.

XIX. The predicate $(\alpha)(E x) T_{1}^{\alpha}(a, a, x)$ of XVIII is a complete predicate of the respective form $(\alpha)(E x) R(a, \alpha, x)$ of (b). Similarly for the other quantified forms of (b).

The hierarchy $\left(b_{2}\right)$ is formally similar to $(a)$. However consider the analog of VIII under this comparison. Half of it holds (by XX for $k=0$ ), but not the converse part (by XXI, since $H_{y}$ for $|y| \geqq \omega$ is nonarithmetical).

$\mathrm{XX}$. For each $k \geqq 0$ : Each predicate arithmetical in predicates expressible in both the $k+1$-function-quantifier forms of (b) (a fortiori, each arithmetical predicate) is expressible in both the $k+1$-functionquantifier forms.

This is proved similarly to XVI (details in $[17,5.2]$ ).

XXI. Each predicate $H_{y}$ for $y \in O$ is expressible in both the 1-function quantifier forms of (b). In fact, there is a primitive recursive function $\tau(y)$ such that, for each $y \in O$,

$$
H_{y}(a) \equiv(\alpha)(E x) T_{1}^{\alpha}\left((\tau(y))_{0}, a, x\right) \equiv(E \alpha)(x) \bar{T}_{1}^{\alpha}\left((\tau(y))_{1}, a, x\right) .
$$

The second sentence of the theorem gives the idea of the proof (details in $[17,8.3]$ ). Predicates definable from recursive predicates by quantification of function variables can also be so defined by quantification of set variables and vice versa $[17,3.8]$. That every predicate $H_{y}$ is definable by such quantification was shown by Davis in [6], for $|y|<\omega^{2}$; and was stated by Mostowski in [23], for $|y|<\omega_{1}$. That one such quantifier suffices is new with this theorem.

6. The predicates expressible in both 1-function-quantifier forms. Since the analogy of (b) to (a) breaks down on VIII, the problem arises of characterizing, independently of the hierarchy (b), the class of the predicates expressible in both the 1 -function-quantifier forms of that hierarchy. By XXI this class contains $H_{y}$ for every $y \in O$, and then by XX every predicate recursive (and hence arithmetical) 
in some $H_{y}$ is in the class. So in [17] we conjectured that the predicates each recursive in $H_{y}$ for some $y \in O$ are the ones expressible in both 1-function-quantifier forms. The principal contribution of the present paper is a proof of this conjecture (cf. XXIV).

By XVII, $(\alpha)(E x) R(a, \alpha, x)$ with $R$ general recursive can be rewritten in the form $(\alpha)(E x) R(a, \bar{\alpha}(x))$, where $R(a, w)$ is primitive recursive, and where in fact (by the definition of $T_{1}^{1}(w, z, a)[16, \mathrm{p}$. 291 ] with [17, Footnote 2]), for each $a$ and $\alpha, R(a, \bar{\alpha}(x))$ is true for at most one $x$.

The number $\bar{\alpha}(x)\left(=\prod_{i<x} p_{i}^{\alpha(t)+1}\right)$ represents the sequence $\alpha(0)$, $\cdots, \alpha(x-1)$ of $x$ numbers. Such a number $\bar{\alpha}(x)$ (for any $\alpha$, and any $x \geqq 0)$ we call a sequence number. The property $\operatorname{Seq}(w)$ of being a sequence number is primitive recursive; in fact, Seq $(w) \equiv w \neq 0$ $\&(i)_{i<\operatorname{lh}(w)}\left[(w)_{i} \neq 0\right]$. The sequence numbers include 1 which represents the empty sequence, and for each $i$ the number $2^{i+1}$ which represents the unit sequence $i$. The sequence $\alpha(0), \cdots, \alpha(x-1)$, $\beta(0), \cdots, \beta(y-1)$ is represented by $\bar{\alpha}(x) * \bar{\beta}(y)$ where $*$ is primitive recursive $[16$, p. 230$]$.

We use $>$ to denote the linear ordering of the sequence numbers which is established by ordering the finite sequences represented by them lexicographically, with shorter sequences coming above longer ones, and using the infinite descending alphabet $\cdots, 2,1,0$. In this ordering, 1 which represents the empty sequence is the highest element. (If $\overline{\operatorname{Seq}}(u)$ or $\overline{\operatorname{Seq}}(v), u>v$ shall be false. The predicate $u>v$ is primitive recursive.)

Now take any predicate $R(a, w)$ and number $a$. We shall say that (with respect to $R$ and $a$ ) a sequence number $w=\bar{\alpha}(x)$ is secured if $(E t)_{t \leqq x} R(a, \bar{\alpha}(t))$, past secured if $(E t)_{t<x} R(a, \bar{\alpha}(t))$, immediately secured if $w$ is secured but not past secured, securable if $w$ is secured or $(\beta)(E t) R(a, \bar{\alpha}(x) * \bar{\beta}(t))$ (i.e. if $(E t) R(a, \bar{\alpha}(t))$ no matter how the values of $\alpha(t)$ for $t \geqq x$ are chosen). ([18, $\S 24]$. If Seq $(w)$, $w$ shall be unsecured and unsecurable. The terms "secured" and "securable" are adapted from Brouwer $[1 ; 2]$.)

Now we define a subset $S_{w}^{R, a}$ of the sequence numbers, for a given sequence number $w$. If $w$ is not past secured, $S_{w}^{R, a}$ shall be the set of all the numbers $w * \bar{\beta}(t)$ which are not past secured. If $w$ is past secured, $S_{w}^{R, a}$ shall be the unit set $\{w\}$. (If $\overline{\operatorname{Seq}}(w), S_{w}^{R, a}$ shall be the empty set. The predicate $u \in S_{w}^{R, a}$ is primitive recursive uniformly in R.)

XXII. Let $\operatorname{Seq}(w)$. The set $S_{w}^{R, a}$ is well-ordered by $>$, if and only if $w$ is securable with respect to $R$ and $a$. So in particular, $S_{1}^{R, a}$ is wellordered by $>$, if and only if $(\alpha)(E x) R(a, \bar{\alpha}(x))$. 
If $w$ is unsecurable, then by definition $w$ is unsecured and $\overline{(\beta)}(E t) R(a, w * \bar{\beta}(t))$, whence $(E \beta)(t) \bar{R}(a, w * \bar{\beta}(t))$. For this $\beta$, $w * \bar{\beta}(t)(t=0,1,2, \cdots)$ is an infinite descending sequence within $S_{w}^{R, a}$. Conversely, given any infinite descending sequence within $S_{w}^{R, a}$, $w$ is unsecured, and by considering that the alphabet $\cdots, 2,1,0$ is well-ordered, we are led to a $\beta$ such that $(t) \bar{R}(a, w * \bar{\beta}(t))$. $[18, \S 26$, especially $(\mathrm{H})$.]

XXIII. Let $R(a, w)$ be primitive recursive, and let Seq(w). There is a primitive recursive function $\xi(a, w)$ such that, if $S_{w}^{R, a}$ is well-ordered, then $\xi(a, w) \in O$ and $|\xi(a, w)|+1$ is the ordinal number of $S_{w}^{R, a}$.

If $S_{w}^{R, a}$ is not $\{w\}$, then

$$
S_{w}^{R, a}=\left(\sum_{i=0,1,2, \ldots} S_{w * 2^{i+1}}^{R, a}\right)+\{w\} .
$$

The sum operation here on the sets is paralleled by a summing of ordinal notations in the construction of $\xi$. $[18, \S 25, \S 26(\mathrm{~F})$.]

XXIV. If $P(a) \equiv(\alpha)(E x) R(a, \alpha, x) \equiv(E \alpha)(x) S(a, \alpha, x)$ with $R$ and $S$ recursive, then for some $y \in O, P(a)$ is general recursive in $H_{y}$.

Proof. By XVII as remarked above, and since $S \equiv \overline{\bar{S}}$ where $\bar{S}$ is also recursive, we can write

$$
P(a) \equiv(\alpha)(E x) R_{0}(a, \bar{\alpha}(x)) \equiv(E \alpha)(x) \bar{R}_{1}(a, \bar{\alpha}(x))
$$

where $R_{j}(j=0,1)$ is primitive recursive, and for a given $a$ and $\alpha$, $R_{j}(a, \bar{\alpha}(x))$ is true for at most one $x$.

If $\alpha$ ranges over all 1-place number-theoretic functions, and we put $\alpha^{i}(x)=(\alpha(x))_{j}$, then $\left(\alpha^{0}, \alpha^{1}\right)$ will range over all pairs $\left(\alpha_{0}, \alpha_{1}\right)$ of 1 -place number-theoretic functions. Let $x-y=x-y$ if $x \geqq y,=0$ if $x<y$. For the present proof, let

$$
w_{j}=\prod_{i<\operatorname{lh}(w)} p_{i}^{\left((w)_{i}-1\right)_{j}+1} .
$$

Then $w_{j}$ is a primitive recursive function of $w$, and $\bar{\alpha}(x)_{j}=\overline{\alpha^{j}}(x)$. Let $R(a, w) \equiv R_{0}\left(a, w_{0}\right) \bigvee R_{1}\left(a, w_{1}\right)$. Let $w_{s t}=w * 2^{2^{s} \cdot 3^{t}+1}$.

By the law of the excluded middle, $(a)[P(a) \vee \bar{P}(a)]$. But

$$
\begin{aligned}
(a) & {[P(a) \vee \bar{P}(a)] } \\
& \equiv(a)\left[(\alpha)(E x) R_{0}(a, \bar{\alpha}(x)) \vee \overline{(E \alpha)}(x) \bar{R}_{1}(a, \bar{\alpha}(x))\right] \\
& \equiv(a)\left[(\alpha)(E x) R_{0}(a, \bar{\alpha}(x)) \vee(\alpha)(E x) R_{1}(a, \bar{\alpha}(x))\right] \\
& \equiv(a)\left(\alpha_{0}\right)\left(\alpha_{1}\right)(E x)\left[R_{0}\left(a, \overline{\alpha_{0}}(x)\right) \vee R_{1}\left(a, \overline{\alpha_{1}}(x)\right)\right] \\
& \equiv(a)(\alpha)(E x)\left[R_{0}\left(a, \bar{\alpha}(x)_{0}\right) \vee R_{1}\left(a, \bar{\alpha}(x)_{1}\right)\right] \equiv(a)(\alpha)(E x) R(a, \bar{\alpha}(x)) .
\end{aligned}
$$

Thus 


$$
(a)(\alpha)(E x) R(a, \bar{\alpha}(x)) .
$$

So by XXII, for every $a$, the set $S_{1}^{R, a}$ is well-ordered by $>$. (The $y$ for the theorem will be a notation for the ordinal number of the sum of the sets $S_{1}^{R, a}$ for $a=0,1,2, \cdots$.)

If $w \in S_{1}^{R, a}$, then either (Case 1 ) $w$ is immediately secured (i.e. $R(a, w)$ ), or else (Case 2$) w$ is unsecured and the numbers $w * 2^{i+1}$ $(i=0,1,2, \cdots)$ and hence in particular the numbers $w_{s t}(s, t$ $=0,1,2, \cdots)$ belong to $S_{1}^{R, a}$ and come below $w$ in the ordering $>$.

Hence we can define predicates $P_{j}(a, w)(j=0,1)$ for all $a$ and for $w \in S_{1}^{R, a}$ by transfinite induction over $S_{1}^{R, a}$ as follows.

$$
\begin{aligned}
& P_{0}(a, w) \equiv \begin{cases}R_{0}\left(a, w_{0}\right) & \text { if } R(a, w), \\
(s)(E t) P_{0}\left(a, w_{s t}\right) & \text { if } \bar{R}(a, w),\end{cases} \\
& P_{1}(a, w) \equiv \begin{cases}R_{1}\left(a, w_{1}\right) & \text { if } R(a, w), \\
(t)(E s) P_{1}\left(a, w_{s}\right) & \text { if } \bar{R}(a, w) .\end{cases}
\end{aligned}
$$

Now we prove that, for each $a$,

$$
P_{0}(a, 1) \vee P_{1}(a, 1) \text {. }
$$

This will follow by setting $w=1$, if we can prove by induction over $S_{1}^{R, a}$ that, for $w \in S_{1}^{R, a}, P_{0}(a, w) \vee P_{1}(a, w)$. CASE 1: $R(a, w)$. By definition, then either $R_{0}\left(a, w_{0}\right)$, in which case $P_{0}(a, w)$, or $R_{1}\left(a, w_{1}\right)$, in which case $P_{1}(a, w)$. CAsE 2: $\bar{R}(a, w)$. Then for each $s$ and $t$, $w_{s t} \in S_{1}^{R, a}$; and by the hypothesis of the induction, $(s)(t)\left[P_{0}\left(a, w_{s t}\right)\right.$ $\left.\vee P_{1}\left(a, w_{s t}\right)\right]$. If $(E s)(t) \bar{P}_{0}\left(a, w_{s t}\right) \&(E t)(s) \bar{P}_{1}\left(a, w_{s t}\right)$, then for the $s$ given by the first and the $t$ by the second we would have $\bar{P}_{0}\left(a, w_{s t}\right)$ $\& \bar{P}_{1}\left(a, w_{s t}\right)$, contradicting the hypothesis of the induction. So $(\overline{E s})(t) \bar{P}_{0}\left(a, w_{s t}\right) \vee(\overline{E t})(s) \bar{P}_{1}\left(a, w_{s t}\right)$, i.e. $(s)(E t) P_{0}\left(a, w_{s t}\right) \bigvee(t)(E s)$ $P_{1}\left(a, w_{s t}\right)$, whence by the definitions of $P_{0}$ and $P_{1}, P_{0}(a, w) \vee P_{1}(a, w)$. Next we prove that, for each $a$,

$$
(E \alpha)(x) \bar{R}_{j}(a, \bar{\alpha}(x)) \rightarrow \bar{P}_{j}(a, 1) \quad(j=0,1) .
$$

Take e.g. $j=0$. Suppose given $a$ and $\alpha$ such that $(x) \bar{R}_{0}(a, \bar{\alpha}(x))$. For this $\alpha$, a sequence number $w$ with $w_{0}=\bar{\alpha}(\mathrm{lh}(w))$ we call an $\alpha$-number. Now by $(1),(\alpha)(E x) R_{1}(a, \bar{\alpha}(x))$. So by XXII, $S_{1}^{R_{1}, a}$ is well-ordered by $>$. We prove by transfinite induction over $S_{1}^{R_{1}, a}$ that for $v \in S_{1}^{R_{1}, a}$, if $w$ is an $\alpha$-number such that $w_{1}=v$, then $\bar{P}_{0}(a, w)$. Since $w$ is an $\alpha$ number, $\bar{R}_{0}\left(a, w_{0}\right)$. CASE $1: R_{1}(a, v)$. Then by $w_{1}=v$ and the definition of $R, R(a, w)$. So by definition, $P_{0}(a, w) \equiv R_{0}\left(a, w_{0}\right)$. So by $\bar{R}_{0}\left(a, w_{0}\right)$, $\bar{P}_{0}(a, w)$. CASE 2: $\bar{R}_{1}(a, v)$. Then for every $t, v * 2^{t+1} \in S_{1}^{R_{1}, a}$. Let $s=\alpha(\operatorname{lh}(w))$. Then for every $t, w_{s t}$ is an $\alpha$-number with $\left[w_{s t}\right]_{1}=v * 2^{t+1}$. So by the hypothesis of the induction, $(t) \bar{P}_{0}\left(a, w_{s t}\right)$. Thus $(E s)(t)$ 
$\bar{P}_{0}\left(a, w_{s t}\right)$, i.e. $\overline{(s)}(E t) P_{0}\left(a, w_{s t}\right)$. By $\bar{R}_{1}(a, v)$ with $w_{1}=v$ and $\bar{R}_{0}\left(a, w_{0}\right)$, $\bar{R}(a, w)$. So by the definition of $P_{0}, \bar{P}_{0}(a, w)$. This completes the induction. But 1 is an $\alpha$-number $w$ with $w_{1}=1$, and $1 \in S_{1}^{R_{1}, a}$. So $\bar{P}_{0}(a, 1)$.

By (4) contraposed and (1),

$$
P_{0}(a, 1) \rightarrow P(a), \quad P_{1}(a, 1) \rightarrow \bar{P}(a),
$$

whence by (3),

$$
P(a) \equiv P_{0}(a, 1) \equiv \bar{P}_{1}(a, 1) .
$$

Now we shall obtain a primitive recursive function $v(a, w)$ such that, for each $a$ and each $w \in S_{1}^{R, a}, P_{0}(a, w)$ as a function of 0 variables (i.e. $\left.\lambda P_{0}(a, w)\right)$ is recursive in $H_{\xi(a, w) * *}$ (cf. XXIII) with Gödel number $v(a, w)$. The property of $v(a, w)$ is to be established by transfinite induction over $S_{1}^{R, a}$. However, instead of defining $v$ first, we shall begin by considering the two cases of the induction, in each case deriving a property of $v$ sufficient to treat the case. Then in conclusion we shall verify that $v$ can be defined so as to possess these properties. CASE $1: R(a, w)$. Then $P_{0}(a, w) \equiv R_{0}\left(a, w_{0}\right)$. So $\lambda a w P_{0}(a, w)$ is recursive, a fortiori recursive in $H_{\xi(a, w) * *}$ say with Gödel number $h_{1}$. So by II* it will suffice to take $v(a, w)=S_{0}^{2,1}\left(h_{1}, a, w\right)$. CASE $2: \bar{R}(a, w)$. Then by the hypothesis of the induction, $\lambda P_{0}\left(a, w_{s t}\right)$ is recursive in $H_{\xi\left(a, w_{s t}\right) * *}$ with Gödel number $v\left(a, w_{s t}\right)$, call this number A. By the definition of $\xi$ in $[18, \S 25], \xi(a, w)=3 \cdot 5^{v}$ where (writing $v_{n}=\Phi_{1}\left(v, n_{o}\right)$ )

$$
v_{n}=\sum_{i<n} o \xi\left(a, w * 2^{i+1}\right) * .
$$

To simplify notation, we write $s t$ for $2^{s} \cdot 3^{t}$ in subscripts of $v$. By the definition of $\sum_{o}$ in $[18, \S 23], v_{s t+1}=v_{s t}+o \xi\left(a, w_{s t}\right)^{*}$; so by $\mathrm{XV}$, $H_{\xi\left(a, w_{s t}\right)^{*}}$ is recursive in $H_{v_{s t+1}}$ with Gödel number $\sigma\left(v_{s t}, \xi\left(a, w_{s t}\right)^{*}\right)$, call it $\mathrm{B}_{1}$. Thence by $\mathrm{X}, H_{\xi\left(a, w_{s t}\right)^{* *}}$ is recursive in $H_{v_{s t+1}}$ with Gödel number $S_{1}^{1,1}\left(g, B_{1}\right)$, call it B. But $v_{s t+1}<_{o} v_{s t+2}$, so $v_{s t+1}{ }^{*} \leqq o v_{s t+2}<o 3 \cdot 5^{v}$ $=\xi(a, w)$; so by XIV, $H_{v_{s t+1}}$ is recursive in $H_{\xi(a, w)}$ with Gödel number $\rho\left(v_{s t+1}{ }^{*}, \xi(a, w)\right)$, call it C. Combining the recursivities with respective Gödel numbers $\mathrm{A}, \mathrm{B}$ and $\mathrm{C}$, we can write $P_{0}\left(a, w_{s t}\right)$ $\equiv G_{2}^{H_{\xi(a, w)}}(u, a, w, s, t)$, where $u$ is a Gödel number of $v$, and $G_{2}^{A}(u, a, w, s, t)$ is partial recursive uniformly in $A$. Thence by two successive applications of the method of proof of VII* (noting that $\lambda s t G_{2}^{A}(u, a, w, s, t)$ is completely defined for the $A, u, a, w$ considered), $(s)(E t) G_{2}^{H \xi(a, w)}(u, a, w, s, t) \equiv \bar{H}_{\xi(a, w) *(}(\theta(u, a, w))$ with a primitive recursive $\theta$. So if $h_{2}$ is a uniform Gödel number from $A$ of $\lambda$ uaw $\bar{A}(\theta(u, a, w))$, then $S_{0}^{3,1}\left(h_{2}, u, a, w\right)$ is one from $H_{\xi(a, w) * *}$ of $\lambda(s)(E t) P_{0}\left(a, w_{s t}\right)$, i.e. of $\lambda P_{0}(a, w)$. So it suffices to take $v(a, w)$ $=S_{0}^{3,1}\left(h_{2}, u, a, w\right)$ for $u$ a Gödel number of $v$. ConcLusion. Let 


$$
\chi(z, a, w)= \begin{cases}S_{0}^{2,1}\left(h_{1}, a, w\right) & \text { if } R(a, w), \\ S_{0}^{3,1}\left(h_{2}, z, a, w\right) & \text { if } \bar{R}(a, w) .\end{cases}
$$

By the cases it suffices to take $v(a, w)=\chi(u, a, w)$ for $u$ a Gödel number of $\chi(u, a, w)$. By III, such a $u$ can be found.

Thence taking $w=1$ and noting $(6), \lambda P(a)$ is recursive in $H_{\xi(a, 1) * *}$ with Gödel number $v(a, 1)$, call it $D$.

We can choose $z$ so that (writing $z_{n}=\Phi_{1}\left(z, n_{o}\right)$ )

$$
z_{n}=\sum_{i<n} o \xi(i, 1)^{*}
$$

$z$ is simply a Gödel number of

$$
\sum_{i<\operatorname{nat}(t)} \xi(i, 1)^{*}
$$

where $\operatorname{nat}(t)=\mu n_{n<t} t=n_{0}$. Let $y=3 \cdot 5^{z}$. Then $y \in O$. Also $z_{a+1}$ $=z_{a}+o \xi(a, 1)^{*}$. So by XV, $H_{\xi(a, 1)^{*}}$ is recursive in $H_{z_{a+1}}$ with Gödel number $\sigma\left(z_{a}, \xi(a, 1)^{*}\right)$, call it $\mathrm{E}_{1}$. Thence by $\mathrm{X}, H_{\xi(a, 1)^{* *}}$ is recursive in $H_{z_{a+1}}$ with Gödel number $S_{1}^{1,1}\left(g, \mathrm{E}_{1}\right)$, call it E. But $z_{a+1}<o z_{a+2}$, so $z_{a+1}{ }^{*} \leqq o z_{a+2}<o y$; so by XIV, $H_{z_{a+1}}$ is recursive in $H_{y}$ with Gödel number $\rho\left(z_{a+1} *, y\right)$, call it $\mathrm{F}$.

Combining the recursivities with Gödel numbers $\mathrm{D}, \mathrm{E}$ and $\mathrm{F}$, we can write $P(a) \equiv G^{H_{\nu}}(a)$ where $G^{A}$ is partial recursive uniformly in $A$. Thus $P(a)$ is general recursive in $H_{y}$.

$\mathrm{XXV}$. The predicate $a \in O$ is expressible in the form $(\alpha)(E x) R(a, \alpha, x)$ with $R$ recursive, and to each recursive predicate $R(a, \alpha, x)$ there is a primitive recursive function $\xi(a)$ such that $(\alpha)(E x) R(a, \alpha, x) \equiv \xi(a) \in O$.

In brief, $a \in O$ is a complete predicate of the form. This is the main result of [18]. To summarize the proof, $a \in O$ can be expressed arithmetically, except for the stipulation that a certain recursive linear ordering is a well-ordering, which can be accomplished by using a universal function quantifier $(\alpha)$. For the second part, we put $\xi(a)=\xi(a, 1)$ after strengthening XXIII to assert also that $\xi(a, w) \in O$ only if $S_{v}^{R, a}$ is well-ordered (by managing to so that $u+o y \in O$ $\rightarrow u, y \in O)$.

In attempting to make the foundations of analysis more constructive, one may attempt in various situations to replace the uncountable infinity of the number-theoretic functions by a countable class of such functions. It is thus of interest to inquire how large a class may be necessary in a given situation. Questions of this kind have been considered by Kreisel $[20 ; 21]$ and the author $[15 ; 17]$. Using terminology suggested by Kreisel, a class $C$ of functions is a basis for a class $D$ of predicates of a function variable, if for every predicate 
$B(\alpha)$ of $D,(E \alpha) B(\alpha) \equiv(E \alpha)[\alpha \in C \& B(\alpha)]$.

Already in the case $D$ consists of the predicates $(x) R(\alpha, x)$ with $R$ recursive, a surprisingly large class $C$ of $\alpha$ 's is necessary. In $[17,5.5]$ we observed that the functions general recursive in 1 -functionquantifier predicates are a basis, while for any fixed $y \in O$ those general recursive in $H_{y}$ are not, but left open the possibility that the functions general recursive in predicates expressible in both 1-function-quantifier forms might be. Now we shall show, as an application of XXIV with XXV, that they are not.

XXVI. There is a recursive predicate $R(\alpha, x)$ such that $(E \alpha)(x) R(\alpha, x)$ is true, but $(x) R(\alpha, x)$ is false for every function $\alpha$ general recursive in predicates $P_{1}, \cdots, P_{l}$ expressible in both the 1-function-quantifier forms of $(\mathrm{b})$.

Proof. We can take the predicates $P_{1}, \cdots, P_{l}$ expressible in both 1 -function-quantifier forms to be one 1-place predicate $P$; in fact, after contracting each $P_{i}$ if necessary to a 1 -place predicate $P_{i}$, by $\mathrm{XX}$ we can use $P(a) \equiv P_{\operatorname{rm}(a, l)+1}([a / l])$ where $a=[a / l] l+\operatorname{rm}(a, l)$ $[16$, p. 223].

The $R(\alpha, x)$ will be $\bar{T}_{1}^{1}(\bar{\alpha}(x), f, f)$ for a number $f$ to be selected.

Consider the predicate

$(E P)(E \alpha)[\{P$ is expressible in both 1-function-quantifier forms $\}$

$\&\{\alpha$ is recursive in $\left.P\} \&(x) \bar{T}_{1}^{1}(\bar{\alpha}(x), a, a)\right] ;$

let it be abbreviated $(E P)(E \alpha) \mathrm{A}(P, \alpha, a)$. Obviously

$$
(E P)(E \alpha) \mathrm{A}(P, \alpha, a) \rightarrow(E \alpha)(x) \bar{T}_{1}^{1}(\bar{\alpha}(x), a, a) .
$$

Assume $\mathrm{A}(P, \alpha, a)$. Then by XXIV, there is a $y$ such that $y \in O$ and $P$ is recursive in $H_{y}$. Then $\alpha$ being recursive in $P$ is also recursive in $H_{y}$, say with Gödel number $e$. Then by $\mathrm{I}^{*}$ (since $\alpha$ is completely defined, as is to be understood), $(i)(E t) T_{1}^{H_{y}}(e, i, t)$. Also $\bar{\alpha}(x)$ $=\prod_{i<x} p_{i} \exp \Phi_{1}^{H_{\nu}}(e, i)+1$, and hence $\bar{T}_{1}^{1}(\bar{\alpha}(x), a, a)$ can be rewritten $(E w)\left[w=\prod_{i<x} p_{i} \exp \Phi_{1}^{H_{y}}(e, i)+1 \& \bar{T}_{1}^{1}(w, a, a)\right]$, which is of the form $(E w) R^{H_{u}}(x, e, a, w)$ where $R^{A}$ is partial recursive uniformly in $A$. By the method of proof of VII*, $(E w) R^{H_{y}}(x, e, a, w) \equiv H_{y^{*}}(\theta(x, e, a))$, with $\theta$ primitive recursive, for values of $y, x, e, a$ which make $\lambda w R^{H}{ }_{v}(x, e, a, w)$ completely defined; but $y \in O$ and $(i)(E t) T_{1}^{H_{y}}(e, i, t)$ guarantee this completeness. Similarly $(E t) T_{1}^{H_{y}}(e, i, t) \equiv H_{y^{*}}(\theta(e, i))$. Altogether, from $\mathrm{A}(P, \alpha, a)$, and hence from $(E P)(E \alpha) \mathrm{A}(P, \alpha, a)$, we thus infer

$$
(E y)(E e)\left[y \in O \&(i) H_{y^{*}}(\theta(e, i)) \&(x) H_{y^{*}}(\theta(x, e, a))\right] ;
$$


write this $(E y)(E e) B(y, e, a)$. Conversely, from $(E y)(E e) B(y, e, a)$ we infer $(E P)(E \alpha) \mathrm{A}(P, \alpha, a)$, by taking for $P$ the predicate $H_{y}$ (cf. XXI) and for $\alpha$ the function recursive in $H_{y}$ with Gödel number $e$.

In $(E y)(E e) B(y, e, a)$, by XXV we can replace $y \in O$ by $(\alpha)(E x)$ $R_{1}(y, \alpha, x)$ with recursive $R_{1}$, and by XXI we can replace $H_{y^{*}}(c)$ by $(\alpha)(E t) T_{1}^{\alpha}\left(\left(\tau\left(y^{*}\right)\right)_{0}, c, t\right)$, after which replacements the resulting expression will reduce by the method of proof of XVI to the form $(\alpha)(E x) R(a, \alpha, x)$ with recursive $R$.

So we have

$$
(E P)(E \alpha) \mathrm{A}(P, \alpha, a) \equiv(\alpha)(E x) R(a, \alpha, x) .
$$

But by XVII, for some number $f$,

$$
(\alpha)(E x) R(a, \alpha, x) \equiv(\alpha)(E x) T_{1}^{1}(\bar{\alpha}(x), f, a) .
$$

Substituting $f$ for $a$ in (1), and in (2) with (3),

$$
\begin{aligned}
& (E P)(E \alpha) \mathrm{A}(P, \alpha, f) \rightarrow(E \alpha)(x) \bar{T}_{1}^{1}(\bar{\alpha}(x), f, f), \\
& (\alpha)(E x) T_{1}^{1}(\bar{\alpha}(x), f, f) \equiv(E P)(E \alpha) \mathrm{A}(P, \alpha, f) .
\end{aligned}
$$

Assuming $(\alpha)(E x) T_{1}^{1}(\bar{\alpha}(x), f, f)$, by (5) and (4) we could infer $(E \alpha)(x) \bar{T}_{1}^{1}(\bar{\alpha}(x), f, f)$, i.e. $\overline{(\alpha)}(E x) T_{1}^{1}(\bar{\alpha}(x), f, f)$. So by reductio ad absurdum, $\overline{(\alpha)}(E x) T_{1}^{1}(\bar{\alpha}(x), f, f)$, i.e. $(E \alpha)(x) \bar{T}_{1}^{1}(\bar{\alpha}(x), f, f)$.

Finally, from $\overline{(\alpha)}(E x) T_{1}^{1}(\bar{\alpha}(x), f, f)$ by $(5)$, we have $\overline{(E P)}(E \alpha)$ $\mathrm{A}(P, \alpha, f)$, which can be rewritten

$(P)(\alpha)[\{\{P$ is expressible in both 1 -function-quantifier forms $\}$ $\&\{\alpha$ is recursive in $\left.P\}\} \rightarrow \overline{(x)} \bar{T}_{1}^{1}(\bar{\alpha}(x), f, f)\right]$.

7. Both 1-function-quantifier forms relative to $Q$. The theorems have versions relativized say to a 1 -place predicate $Q$. For $\psi$ the representing function of $Q$, we write $T_{1}^{Q, \alpha}(z, a, x) \equiv T_{1}^{1,1}(\Psi(x), \bar{\alpha}(x), z, a)$ $\equiv T_{1}^{Q, 1}(\bar{\alpha}(x), z, a)$. Using functions partial recursive in $Q$ instead of partial recursive absolutely, we obtain a system of notation for ordinals constructive in $Q$, with notions $O^{Q},<_{0}^{Q}, \| Q, \omega_{1}^{Q},+_{0}^{1}, \sum_{0}^{1}$ in place of $O,<_{o}, \|, \omega_{1},+_{o}, \sum_{o}[17,6.4 ; 18, \S 30]$; the superscript for $+_{0}^{1}, \sum_{0}^{1}$ is " 1 " rather than "Q" because only the number 1 of the arguments of $Q$ matters. Now we define $H_{1}^{Q}(a) \equiv Q(a) ; H_{2^{y}}^{Q}(a)$ $\equiv(E x) T_{1}^{Q, H_{y}^{Q}}(a, a, x)$ if $y \in O^{Q}$; and $H_{3 \cdot 5^{y}}^{Q}(a) \equiv H_{y}^{Q} Q_{(a)}\left((a)_{0}\right)$ if $3 \cdot 5^{y} \in O^{Q}$ and $y_{n}^{Q}=\Phi_{1}^{Q}\left(y, n_{0}\right)$. In the case of a statement such as " $A$ is recursive in $B$ " which is already relative, the direct relativization to $Q$ is " $A$ is recursive in $Q, B$ ". However in most such cases to be considered, $Q$ will be recursive in the $B$, so " $Q$," need not be inserted. We have inserted the superscript "Q," on the " $T_{1}$ " in the definition of $H_{2^{y}}^{Q}$ be- 
cause the proofs are easier using the direct relativization, although the degrees of the predicates $H_{y}^{Q}$ would be the same without it $[17,6.8]$.

XXVII. Theorems XIV-XXVI hold when in place of the notions 'recursive', ' $T_{1}^{\alpha}$ ', ' $T_{1}^{1}$,' ' $T_{1}^{\alpha, \beta}$, 'arithmetical', 'analytic', ' $k$-functionquantifier forms', 'O', '<o', ' $\|^{\prime}$ ', '十o', ' $H_{y}$ ', ' $\rho$ ', ' $\sigma$ ', ' $\tau$ ', ' $\xi$ ' we use 'recursive in $Q$ ', ' $T_{1}^{Q, \alpha}$, ' $T_{1}^{Q, 1}$, ' ' $T_{1}^{Q, \alpha, \beta}$ ', 'arithmetical in $Q$ ', 'analytic in $Q$ ', ' $k$-function-quantifier forms relative to $Q$ ', ' $O^{Q}$ ', ' $<_{0}^{Q}$, , ' ||$Q^{\prime}$, ' $+{ }_{0}^{1}$, ' $H_{y}^{Q}$ ', ' $\rho^{1}$ ', ' $\sigma^{1}$ ', ' $\tau^{1}$ ', ' $\xi^{Q}$ ', respectively, except that $\rho^{1}, \sigma^{1}, \tau^{1}$ are primitive recursive simply, in XIV $\rho^{1}(u, y)$ is a Gödel number of $H_{u}^{Q}$ from $Q, H_{y}^{Q}$ (and similarly $\sigma^{1}$ in $\mathrm{XV}$ ) but also there is a function $\rho^{Q}(u, y)$ partial recursive uniformly in $Q$ which gives a Gödel number of $H_{u}^{Q}$ from $H_{y}^{Q}$ simply, and in XXIV we use recursiveness simply in $H_{y}^{Q}$ (call them then $\mathrm{XIV}^{*}$-XXVI*).

Proof. For XIV*, cf. $[17,6.6]$. For XXIV*, we relativize the proof of XXIV directly, using $\rho^{1}, \sigma^{1}$ and a primitive recursive function $v^{1}(a, w)$ which gives a Gödel number of $\lambda P_{0}^{Q}(a, w)$ from $Q, H_{\xi^{Q}(a, w)^{* *} \text {. }}^{Q}$ Thus we establish that the $P(a)$ is recursive in $Q, H_{y}^{Q}$ for some $y \in O^{Q}$; but by the version of $\mathrm{XIV}^{*}$ with $\rho^{Q}(u, y), Q$ is recursive in $H_{y}^{Q}$ (with Gödel number $\rho^{Q}(1, y)$ ).

Let us say a predicate $P$ is hyperarithmetical (hyperarithmetical in $Q)$, if $P$ is recursive in $H_{y}$ for some $y \in O\left(H_{y}^{Q}\right.$ for some $\left.y \in O^{Q}\right)$, or equivalently by XX, XXI and XXIV (XX*, XXI*, and XXIV*) if $P$ is expressible in both 1 -function-quantifier forms (both 1-functionquantifier forms relative to $Q$ ). We choose the name because the predicates recursive in $H_{y}$ for some $y \in O$ constitute the extended arithmetical hierarchy.

The relation ' $P$ is hyperarithmetical in $Q$ ' is obviously reflexive; and it is transitive, by use of the case of $\mathrm{XX}^{*}$ for $k=0$ and with a free function variable $\alpha[17,5.2]$, followed by contraction of adjacent like function quantifiers $(\alpha)(\beta)$ or $(E \alpha)(E \beta)$. Hence ' $P$ is hyperarithmetical in $Q$ and vice versa' is reflexive, symmetric and transitive, and gives rise to a notion of hyperdegree analogous to the notion 'degree' for ' $P$ is recursive in $Q$ and vice versa'. The hyperdegree of $P$ is less than that of $Q$, if $P$ is hyperarithmetical in $Q$ but not vice versa. Predicates of the same degree are a fortiori of the same hyperdegree. If $P$ is of lower degree than $Q$, a fortiori it is of the same or lower hyperdegree. A predicate hyperarithmetical in a hyperarithmetrical predicate is hyperarithmetical (using $\mathrm{XX}$ with free $\alpha$ and contraction).

If $A$ is hyperarithmetical in $B$, and $R^{A}$ is recursive in $A$, then $(\alpha)(E x) R^{A}(a, \alpha, x)$ is expressible in the form $(\alpha)(E x) R^{B}(a, \alpha, x)$ with $R^{B}$ recursive in $B$ (using $\mathrm{XX}^{*}$ with free $\alpha$ and contraction). Hence if 
further $\mathbb{C}^{B}$ is a complete predicate of the form $(\alpha)(E x) R^{B}(a, \alpha, x)$ (cf. VII, $\left.\mathrm{XIX}^{*}\right)$, then $(\alpha)(E x) R^{A}(a, \alpha, x)$ is recursive in $\mathbb{S}^{B}$. Applying this in two directions to predicates $Q_{1}, Q_{2}$ of the same hyperdegree,

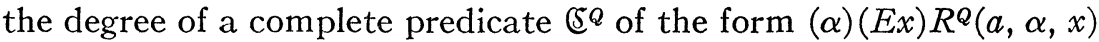
with $R^{Q}$ recursive in $Q$ (which is the maximal degree for predicates of the form) depends only on the hyperdegree of $Q$. The degree and hyperdegree of $\mathbb{S}^{Q}$ are greater than those of $Q$ (using XVIII*).

Using 'hyperarithmetical' instead of 'arithmetical', not only does the analog of VIII come to hold (cf. the remarks preceding XX), but also the following analog of half of XI holds.

XXVIII. For each $k \geqq 0$ : The predicates hyperarithmetical in predicates expressible in the k-function-quantifier forms of (b) are expressible in both the $k+1$-function-quantifier forms.

Using XX with free $\alpha$ and contraction. Similarly we have a XXVIII* for the forms relative to $Q$.

At the present writing we do not know whether the analog of the converse part of XI holds. Consequently we do not know whether the hierarchies analogous to the two of XII are equivalent with respect to degrees (after their first two members). Thus let $\mathfrak{N}_{0}(a)$ $\equiv \Omega_{0}(a) \equiv a=a$; let $\mathfrak{R}_{1}, \mathfrak{R}_{2}, \ldots$ be the predicates of the upper row of XVIII; and let $\mathfrak{\Omega}_{k+1}(a) \equiv(\alpha)(E x) T_{1}^{\ell_{k, \alpha}}(a, a, x)$.

XXIX. For each $k \geqq 0: \mathfrak{N}_{k+1}$ is expressible in a $k+1$-function-quantifier form of (b), is of maximal degree for predicates so expressible, and is of higher degree and hyperdegree than $\mathfrak{N}_{k} . \mathfrak{R}_{k+1}$ is expressible in a $k+1$-function-quantifier form of (b), and also in the form $(\alpha)(E x)$ $R^{\mathfrak{R}_{k}}(a, \alpha, x)$ with $R^{\mathcal{R}}$. recursive in $\mathfrak{R}_{k}$, is of maximal degree for predicates expressible in the latter form, and is of higher degree and hyperdegree than $\mathfrak{R}_{k}$. (So $\mathfrak{R}_{k+1}$ is of degree $\leqq$ the degree of $\mathfrak{R}_{k+1}$.)

An XXIX* refers similarly to XVIII* $\left(\Re_{0}^{Q}(a) \equiv \Omega_{0}^{Q}(a) \equiv Q(a)\right.$, etc. $)$.

$\mathrm{XXX}$. There is a primitive recursive function $\nu^{1}(a)$ such that, for any 1-place predicate $Q, Q(a) \equiv \nu^{1}(a) \in O^{Q}$.

We make $\nu^{1}(a)$ a notation for $\omega$ exactly when $Q(a)[17,6.4]$.

By XXV, $a \in O$ is of maximal degree for predicates $(\alpha)(E x) R(a, \alpha, x)$ with $R$ recursive. By XXX, $Q$ is recursive in $a \in O^{Q}$; so by $\mathrm{XXV}^{*}$, $a \in O^{Q}$ is of maximal degree for predicates $(\alpha)(E x) R^{Q}(a, \alpha, x)$ with $R^{Q}$ recursive in $Q$. The degree of $a \in O^{Q}$ depends only on (and the hyperdegree of $H_{y}^{Q}$ for any $y \in O^{Q}$ is) the hyperdegree of $Q$; for $Q$ hyperarithmetical, the degree of $a \in O^{Q}$ is that of $a \in O$ (and $H_{y}^{Q}$ for $y \in O^{Q}$ is hyperarithmetical, i.e. of minimal hyperdegree). Now $a \in O, a \in O^{o}$ (where $O^{o}=O^{\lambda a} \in o$ ), $a \in O^{o^{o}}$ (where $O^{o}=O^{\lambda a a \in O^{o}}$ ), .. are of the same respective degrees as $\mathfrak{R}_{1}, \mathfrak{R}_{2}, \mathfrak{R}_{3}, \cdots$.

Instead of successive systems of notations for ordinals of the first 
and second number classes $O, O^{\circ}, O^{\circ}, \cdots$, the first constructive and the others each constructive in the predicate of being a notation of the preceding system, we can introduce higher constructive number classes in the manner indicated in Church-Kleene [5]. The relations between these two ways of extending the representation of ordinals (cf. [17, end 6.4] and Spector [28, \$8]) and the correlation of predicates to ordinals are being investigated.

Hierarchies can be set up similarly to the analytic hierarchy but using function variables of higher finite types (at least). For these we have established the analogs of a number of the theorems considered above for the arithmetical and analytic hierarchies. We plan to discuss these hierarchies in a paper to be entitled "Analytic predicates and function quantifiers of higher finite types." That new number-theoretic predicates are definable by use of successively higher finite types of variables was already known from Tarski [29].

Mostowski [22] compared the arithmetical hierarchy with the hierarchy of projective sets studied by Lusin and others. Under his comparison the analogy was imperfect (cf. our [14]). There are further possibilities for the utilization of analogies in this direction, which J. W. Addison, Jr. plans to discuss.

\section{BIBLIOGRAPHY}

1. L. E. J. Brouwer, Beweis, dass jede volle Funktion gleichmässig stetig ist, Neder. Akad. Wetensch. vol. 27 (1924) pp. 189-193. $60-75$.

2. —- Über Definitionsbereiche von Funktionen, Math. Ann. vol. 97 (1927) pp.

3. A. Church, $A n$ unsolvable problem of elementary number theory, Amer, J. Math. vol. 58 (1936) pp. 345-363.

4. - The constructive second number class, Bull. Amer. Math. Soc. vol. 44 (1938) pp. 224-232.

5. A. Church and S. C. Kleene, Formal definitions in the theory of ordinal numbers, Fund. Math. vol. 28 (1936) pp. 11-21.

6. M. Davis, On the theory of recursive unsolvability, Thesis, Princeton, 1950.

7. - Relatively recursive functions and the extended Kleene hierarchy, Proceedings of the International Congress of Mathematicians, Cambridge, Mass., 1950, Providence, American Mathematical Society, 1952, vol. 1, p. 723.

8. K. Gödel, Über formal unentscheidbare Sätze der Principia Mathematica und verwandter Systeme I, Monatshefte für Mathematik und Physik vol. 38 (1931) pp. 173198.

9. - On undecidable propositions of formal mathematical systems, Notes on lectures at the Institute for Advanced Study, mimeographed, Princeton, 1934, $30 \mathrm{pp}$.

10. S. C. Kleene, General recursive functions of natural numbers, Math. Ann. vol. 112 (1936) pp. 727-742.

11. - On notation for ordinal numbers, J. Symbolic Logic vol. 3 (1938) pp. 150-155.

12. - Recursive predicates and quantifiers, Trans. Amer. Math. Soc. vol. 53 
(1943) pp. 41-73. Section 15 is affected by the error in [13].

13. - On the forms of the predicates in the theory of constructive ordinals Amer. J. Math. vol. 66 (1944) pp. 41-58. An error in this paper is pointed out in $[15$, p. $685 ; 16$, p. 527], and a corrected treatment will appear in [18].

14. —, A symmetric form of Gödel's theorem, Neder. Akad. Wetensch. vol. 53 (1950) pp. 800-802 (also Indagationes Mathematicae vol. 12 (1950) pp. 244-246).

15. - Recursive functions and intuitionistic mathematics, Proceedings of the International Congress of Mathematicians, Cambridge, Mass., 1950, Providence, American Mathematical Society, 1952, vol. 1, pp. 679-685.

16. - Introduction to metamathematics, New York, Van Nostrand, Amsterdam, North Holland, and Groningen, Noordhoff, 1952, 10+550 pp.

17. - Arithmetical predicates and function quantifiers, to appear in Trans. Amer. Math. Soc.

18. - On the forms of the predicates in the theory of constructive ordinals (second paper), to appear in Amer. J. Math.

19. S. C. Kleene and E. L. Post, The upper semi-lattice of degrees of recursive unsolvability, Ann. of Math. (2) vol. 59 (1954) pp. 379-407.

20. G. Kreisel, On the interpretation of non-finitist proofs, J. Symbolic Logic vol. 16 (1951) pp. 241-267 and vol. 17 (1952) pp. 43-58.

21. - A variant to Hilbert's theory of the foundations of arithmetic, Brit. J. Philos. Sci. vol. 4 (1953) pp. 107-129.

22. A. Mostowski, On definable sets of positive integers, Fund. Math. vol. 34 (1946) pp. 81-112.

23. - A classification of logical systems, Studia Philosophica vol. 4 (1951) pp. 237-274.

24. E. L. Post, Finite combinatory processes-formulation I, J. Symbolic Logic vol. 1 (1936) pp. 103-105.

25. - Recursively enumerable sets of positive integers and their decision problems, Bull. Amer. Math. Soc. vol. 50 (1944) pp. 284-316.

26. - Degrees of recursive unsolvability, Bull. Amer. Math. Soc. Abstract 54-7-269.

27. T. Skolem, Über die Zurïckführbarkeit einiger durch Rekursionen definierten Relationen auf "arithmetische," Acta Sci. Math. Szeged vol. 8 (1936-37) pp. 73-88.

28. C. Spector, Recursive well-orderings, to appear in J. Symbolic Logic.

29. A. Tarski, Einige Betrachtungen über die Begriffe der w-Widerspruchsfreiheit und der $\omega$-Vollständigkeit, Monatshefte für Mathematik und Physik vol. 40 (1933) pp. 97-112.

30. A. M. Turing, On computable numbers, with an application to the Entscheidungsproblem, Proc. London Math. Soc. (2) vol. 42 (1936-37) pp. 230-265. A correction, ibid. vol. 43 (1937) pp. 544-546.

31. - Systems of logic based on ordinals, Proc. London Math. Soc. (2) vol. 45 (1939) pp. 161-228.

\section{UNIVERSITY OF WISCONSIN}

